\section{Noncredible Symptom History}

Nicholas Pastorek

Polytrauma Planning Director, Rehabilitation and Extended Care Line, Michael E. DeBakey VA Medical Center, Houston, TX, USA discrepancy between reported symptom severity and objective findings should raise suspicion for noncredible presentation. Review of available medical records and collateral information from a reliable third party is imperative when investigating symptom credibility.

\section{See Also}

Inaccurate symptom reporting that presents a serious threat to diagnostic validity in medical contexts, especially where diagnoses are based primarily on self-report of injury history and/or symptoms. Noncredible symptom history is most commonly encountered in evaluation contexts where external incentives (e.g., financial gain, mitigation of responsibility for misconduct) are present. Atypical chronology of symptom onset, unusual symptom pattern, marked changes in injury history/symptom report over time, and
Malingering

- Symptom Validity Assessment

\section{References and Readings}

Boone, K. B. (2007). Assessment of feigned cognitive impairment. New York: The Guilford Press.

Larrabee, G. J. (2007). Assessment of malingered neuropsychology deficits. New York: Oxford University Press. 